\title{
PROPRIEDADE INTELECTUAL NA PÓS-GRADUAÇÃO DAS UNIVERSIDADES FEDERAIS DO NORDESTE: INDICADORES BIBLIOMÉTRICOS
}

INTELLECTUAL PROPERTY IN THE POST GRADUATION OF NORTHEAST FEDERAL UNIVERSITIES: BIBLIOMETRIC INDICATORS

1 Jandira Reis Vasconcelos

'João Antonio Belmino dos Santos

Universidade Federal de Sergipe ${ }^{1}$

\section{Correspondência}

Priscila Machado Borges Sena

Universidade Federal de Sergipe

São Cristóvão - SE - Brasil

E-mail: jandirasantosreis@ yahoo.com.br

\section{Submetido em: 27/06/2018}

Aceito em: 14/11/2018

Publicado em: 06/12/2018

Checagem antiplágio

turnitind

JITA: BB. Bibliometric methods

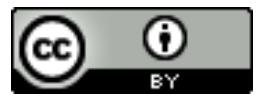

e-Location: e019007 


\section{RDBCI

\section{RESUMO}

A análise da produção do conhecimento científico, através da bibliometria, permite avaliar o perfil de área específica. Neste sentido, este artigo apresenta os indicadores bibliométricos dos Programas de Pós-Graduação da região Nordeste que pesquisam sobre Propriedade Intelectual. Esta pesquisa classifica-se como exploratória, com abordagens quantitativa e qualitativa, tendo como campo de estudo: Universidade Federal da Bahia (UFBA), Universidade Federal de Sergipe (UFS), Universidade Federal de Alagoas (UFAL), Universidade Federal de Pernambuco (UFPE), Universidade Federal da Paraíba (UFPB), Universidade Federal do Ceará (UFC), Universidade Federal do Maranhão (UFMA), Universidade Federal do Piauí (UFPI), Universidade Federal do Rio Grande do Norte (UFRN). A coleta de dados foi feita na Base de Dados de Teses e Dissertações da Coordenação de Aperfeiçoamento de Pessoal de Nível Superior (CAPES) e nos Repositórios Institucionais das instituições pesquisadas. Como estratégia de busca, utilizou-se o termo "Propriedade Intelectual", que permitiu encontrar 4 (quatro) teses e 34 dissertações. Para organização dos dados foram utilizados os softwares Excel ${ }^{\circledR}$ e Word Art®. Com os resultados, foi possível identificar os Programas de Pós-Graduação que desenvolveram pesquisas em Propriedade Intelectual, as temáticas abordadas e as palavras-chave mais recorrentes. Dessa forma, foi viável traçar um panorama dos aspectos da pesquisa em Propriedade Intelectual através da construção de indicadores bibliométricos.

\section{PALAVRAS-CHAVE}

Propriedade intelectual. Bibliometria. Pós-Graduação.

\section{ABSTRACT}

The analysis of the production of scientific knowledge through bibliometrics allows the evaluation of the specific area profile. In this sense, this article presents the bibliometric indicators of the Postgraduate Programs of the Northeast region that research on intellectual property. This research is classified as exploratory, with quantitative and qualitative approaches, having as a field of study: Federal University of Bahia (UFBA), Federal University of Sergipe (UFS), Federal University of Alagoas (UFAL), Federal University of Pernambuco (UFPE), Federal University of Paraíba (UFPB), Federal University of Ceará (UFC), Federal University of Maranhão (UFMA), Federal University of Piauí (UFPI), Federal University of Rio Grande do Norte (UFRN). The data collection was done in the CAPES Thesis and Dissertations Database and in the Institutional Repositories of the researched institutions. As a search strategy the term "Intellectual Property" was used, which allowed to find 4 theses and 34 dissertations. To organize the data, Excel and Word Art software were used. With the results, it was possible to identify the Graduate Programs that developed research on Intellectual Property, the topics addressed and the most recurrent keywords. Thus, it was feasible to outline the aspects of Intellectual Property research through the construction of bibliometric indicators.

\section{KEYWORDS}

Intellectual property. Bibliometria. Postgraduate studies. 


\section{RPBO $\begin{aligned} & \text { Revista Digital Biblioteconomia e Ciência da } \\ & \text { Informação }\end{aligned}$ P B B

\section{Introdução}

A análise da produção científica resultante dos programas de Pós-Graduação tem sido objeto de estudo em diversos campos do conhecimento (CUSTÓDIO, 2012). Estudos dessa natureza permitem verificar as tendências de áreas, temáticas, instituições, pesquisadores, redes de colaboração, entre outros elementos.

Com o aumento da Pós-Graduação, consequentemente, houve o crescimento da produção científica, por sua vez, gerando novos conhecimentos em áreas diversas. Entre as pesquisas produzidas no meio acadêmico, sobretudo nos Programas de Pós-Graduação, temse teses e dissertações, que são valiosos instrumentos de comunicação científica, avaliados pelos pares.

Neste sentido, as pesquisas com abordagens bibliométricas em teses e dissertações podem contribuir para identificar as tendências teóricas, sobre o tema estudado, identificar qual a colaboração dos Programas de Pós-Graduação para determinada área do conhecimento, delinear tendências metodológicas, bem como identificar autores mais citados. Tais informações podem se tornar relevantes para pesquisadores e grupos de pesquisas.

As pesquisas desenvolvidas nas universidades sejam elas acadêmicas, básicas ou aplicadas, visam, sobretudo produzir novos conhecimentos. No âmbito da Pós-Graduação, em nível de mestrado e doutorado, a divulgação da produção científica pode ser feita através das teses e dissertações, bem como em periódicos científicos. Estes documentos registram o conhecimento novo, são credíveis, visto que são avaliados pelos pares e dão visibilidade aos resultados das pesquisas (CUSTÓDIO, 2012). Não basta apenas descobrir e construir novos conhecimentos, é necessário também ter informações de como, quando e onde um conhecimento pode ser protegido, de forma a gerar novos produtos.

É neste contexto que se insere a Propriedade Intelectual, devido a sua importância no meio acadêmico, pois tem como premissa a proteção da criação humana, seja no campo literário, artístico ou científico. Alicerçada no contexto jurídico e com relevância no fator de competitividade no mercado, sua divulgação é pertinente para todas as áreas do conhecimento. Com o advento da Lei de Inovação em 2004, a Propriedade Intelectual passou a ter mais relevância nas universidades, devido à formação dos Núcleos de Inovação e Tecnologia (NIT’s), que são as entidades responsáveis pelas questões relativas ao licenciamento, proteção e transferência das inovações tecnológicas.

Dessa forma, o objetivo desse trabalho é fazer uma análise bibliométrica, nas teses e dissertações que abordam a Propriedade Intelectual, visando contribuir com indicadores bibliométricos da produção científica nesta temática, desenvolvida por pesquisadores dos

\begin{tabular}{l|l|l|l|l|l}
\hline (c) RDBCl: Rev. Digit. Bibliotecon. Cienc. Inf. & Campinas, SP & v.17 & $1-23$ & e019007 & 2018
\end{tabular}




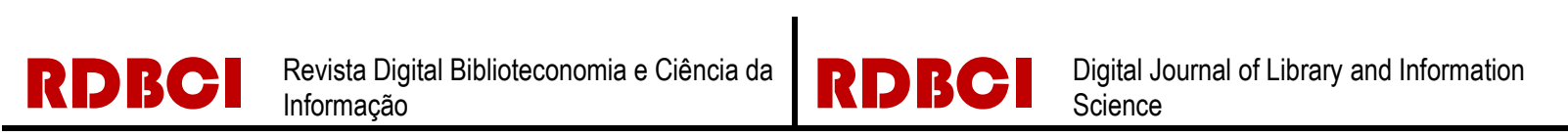

Programas de Pós-Graduação das universidades federais da região Nordeste. Objetiva especificamente identificar os Programas de Pós-Graduação que estão pesquisando, as áreas de conhecimento que estão relacionadas, bem como as temáticas que estão sendo mais abordadas nas pesquisas em Propriedade Intelectual. Neste sentido, esta pesquisa, justifica-se pela importância de compreender a dinâmica da Propriedade Intelectual com outros campos do conhecimento, bem como contribuir para pesquisas futuras em estudos bibliométricos e contribuir com indicadores da produção científica sobre a Propriedade Intelectual.

Os tópicos seguintes a esta introdução contemplam o referencial teórico, que aborda bibliometria, indicadores bibliométricos e a Propriedade Intelectual. Após o referencial teórico apresenta-se a metodologia, seguida dos resultados e discussão, finalizando com as considerações finais.

\section{Bibliometria e Indicadores Bibliométricos}

A bibliometria diz respeito à avaliação da produção do conhecimento científico, tendo surgido no século XX como uma técnica utilizada para mensurar quantitativamente os índices da produtividade científica (LOPES et al., 2012). Apesar de ser amplamente utilizada pela Biblioteconomia e pela Ciência da Informação, pesquisadores de diversas áreas do conhecimento empregam a bibliometria com a finalidade de explorar o impacto da ciência, no que diz respeito à produção e divulgação, bem como à construção de indicadores (MARCELO; HAYASHI, 2013).

De acordo com Gorbea Portal (2016), como especialidade métrica, a bibliometria permite identificar as regularidades quantitativas existentes no fluxo de informação documental e nos processos de produção e comunicação científica que operam nestes. Para Araújo (2006), a bibliometria contempla a análise de livros, periódicos científicos, artigos de periódicos e teses, entre outras fontes. Possibilita medir índices de produção e disseminação do conhecimento, bem como avaliar a produtividade dos autores e realizar estudos de citações por meio de indicadores.

Como contribuição aos estudos da análise bibliométrica, tem-se as leis clássicas da bibliometria, que norteiam os pesquisadores quanto a categorizar o foco de estudo e a aplicabilidade. Guedes (2012) mostra os autores das leis e suas relevâncias: a Lei de Bradford está voltada à análise da produtividade de periódicos, permite analisar a relevância de periódicos em determinada área do conhecimento. A Lei de Lotka se relaciona aos estudos da produtividade científica dos autores, enfatiza que os pesquisadores de maior influência produzem mais, e os pesquisadores com menos influência produzem menos em dada área do conhecimento. Na Lei de Zipf, tem-se a possibilidade de avaliar a ocorrência de frequência de

v.17

$1-23$

e019007
2018 


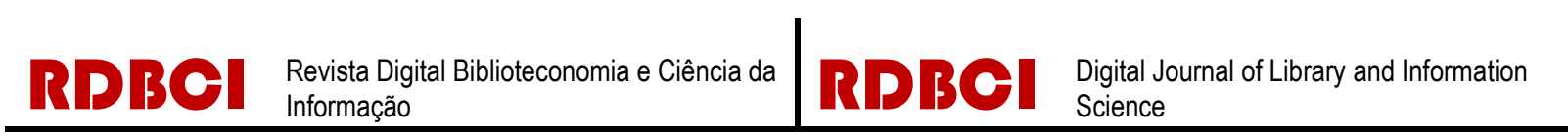

determinada palavra em um texto, estando relacionada à indexação temática.

No que diz respeito aos indicadores bibliométricos, Lopes et al. (2012) classificam em: Indicadores de qualidade científica, referentes à avaliação do conteúdo pelos pares; Indicadores de atividade científica, relacionados à quantificação das atividades desenvolvidas; Indicadores de associações temáticas, que são as análises de citações e de referências; Indicadores de impacto científico, os quais se dividem em dois tipos, os indicadores de impacto dos trabalhos, voltado à análise do número de citações recebidas e o outro tipo concernente aos indicadores de impacto das fontes, que analisam o fator de impacto dos periódicos, o índice de citações e a influência das revista. De acordo com Mugnaini (2018),apesar da constatação da importância da avaliação da pesquisa científica, os indicadores de impacto (baseados em citação) são compreendidos como complementares, não descartando a necessidade de uma investigação mais exaustiva.

A análise do conhecimento científico por meio de indicadores confiáveis permite verificar o crescimento de diferentes áreas do conhecimento, a visibilidade da produção científica, dos autores, instituições e países. Seus resultados podem auxiliar na tomada de decisões para investimento em ciência e tecnologia (ALVAREZ; CAREGNATO, 2017).

É evidente o quanto a bibliometria traz de possibilidades para avaliação da ciência. Pimenta et al. (2017) destacam que a bibliometria é de fundamental importância para a investigação da produção científica em virtude de seus atributos, pois enseja apontar métricas de estudos, identificar redução de temas, medir impacto de citação, bem como verificar a produção científica no meio acadêmico. Possibilita ainda a visibilidade das novas fontes de informações, através de análise em patentes, teses, dissertações e demais publicações da pesquisa científica.

\subsection{Propriedade intelectual: definição e abrangência}

De acordo com a convenção da Organização Mundial da Propriedade Intelectual (OMPI), a Propriedade Intelectual (PI) diz respeito a toda criação da mente humana, em todas as áreas do conhecimento, seja no meio científico, tecnológico, artístico ou literário. Versa sobre o direito de proteção das invenções em todos os domínios da atividade humana, dando ao inventor as garantias e possibilidades de recompensas pelo reconhecimento da criação (WIPO, 2016). Campos e Denig (2011) complementam que os resultados das atividades intelectuais são reconhecidos como ativos intangíveis, ou seja, ativos baseados em conhecimento.

Para Speziali et al. (2016) a PI caracteriza-se como instrumento legal, de tutela ao conhecimento proveniente da criação humana, nos aspectos tecnológicos, artísticos e

\begin{tabular}{l|l|l|l|l|l}
\hline (c) RDBCl: Rev. Digit. Bibliotecon. Cienc. Inf. & Campinas, SP & v.17 & $1-23$ & e019007 & 2018
\end{tabular}




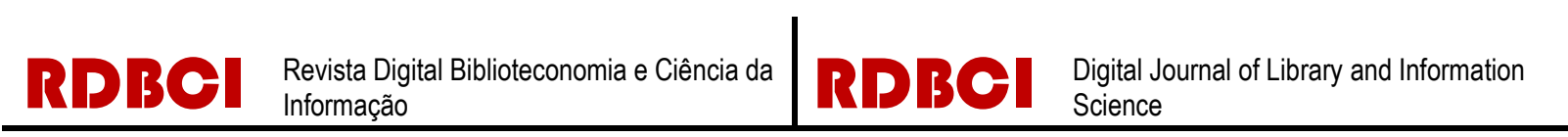

literários. Além disso, pode estimular o avanço socioeconômico dos países, caso possua uma gestão de PI bem-sucedida, bem como impactar na dinâmica da economia das nações.

Quanto à abrangência, a PI engloba três grandes áreas: o direito autoral, a Propriedade Industrial e a proteção sui generis. Cada um desses segmentos dispõe de legislação específica quanto a sua forma de proteção, por possuírem naturezas distintas (UFS, 2017; JUNGMANN; BONETTI, 2010).

O direito autoral é regulamentado pela Lei $n^{\circ} 9.610 / 98$, consiste na proteção de obras intelectuais no campo literário, científico e artístico, tais como: desenhos, pinturas, esculturas, livros, conferências, artigos científicos, matérias jornalísticas, músicas, filmes, fotografias, software, entre outros (BRASIL, 1998). Contempla o direito de autor, direitos conexos e programa de computador.

A Propriedade Industrial, regulamentada pela Lei 9279/96, tem um foco maior na atividade industrial, tecnológica e comercial (SPEZIALI et al., 2016). Envolve os direitos sobre as patentes de invenção, os modelos industriais, as marcas, a repressão à concorrência desleal.

Proteção sui generis, área de proteção menos conhecida na Propriedade Intelectual, envolve a topografia de circuito integrado, cultivar, conhecimentos tradicionais e o acesso ao patrimônio genético, sendo cada tipo de proteção regulamentada por lei específica, bem como a determinação dos prazos de proteção. (UFS, 2017; JUNGMANN; BONETTI, 2010).

\section{Método}

Esta pesquisa classifica-se como exploratória, com abordagens qualitativa e quantitativa. Quanto aos procedimentos adotados, a pesquisa baseou-se na técnica bibliométrica, tendo em vista que permite a análise em livros, periódicos científicos, artigos de periódicos, teses, dissertações entre outros, por meio de técnicas da estatística e da matemática, para construção de indicadores que representem a dinâmica de determinada área do conhecimento científico (ARAÚJO, 2006; LOPES, 2012; MARCELO; HAYASHI, 2013).

O campo de estudo foi formado por nove Universidades Federais da Região Nordeste do Brasil, a seguir: UFAL, UFBA, UFC, UFMA, UFPB, UFPE, UFPI, UFRN e UFS.

Quanto aos instrumentos de coletas dos dados, foram coletados através do acesso ao site eletrônico da Biblioteca Digital Brasileira de Teses e Dissertações (BDTD) do Instituto Brasileiro de Informação em Ciência e Tecnologia (IBICT) e dos Repositórios

\begin{tabular}{l|l|l|l|l|l|}
\hline C RDBCl: Rev. Digit. Bibliotecon. Cienc. Inf. & Campinas, SP & v.17 & $1-23$ & e019007 & 2018 \\
\hline
\end{tabular}




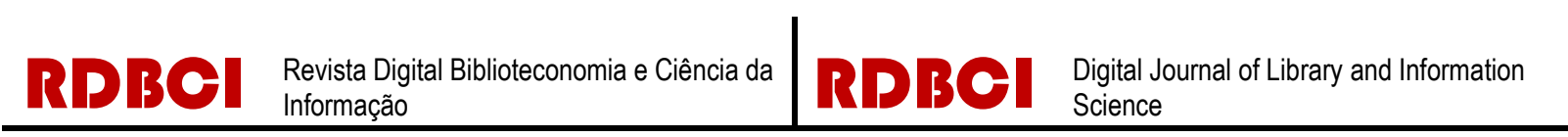

Institucionais (RI). A escolha dessas fontes se deu pelo fato das mesmas disponibilizarem informações científicas atualizadas, com abrangência nacional.

A BDTD foi desenvolvida e é coordenada pelo IBICT, tem como objetivo integrar os sistemas de informação de teses e dissertações das instituições de ensino superior, e disponibilizar aos pesquisadores um catálogo com texto integral. Os dados são fornecidos pelas instituições de defesa, que também possuem as suas BDTD (IBICT, 2018).

Os RIs foram desenvolvidos pelo projeto IBICT-FINEP/PCAL/XBDB. São bases de dados online que reúnem a produção científica de uma instituição, proporcionando maior visibilidade aos resultados de pesquisas e possibilitam a preservação da memória científica de sua instituição (IBICT, 2018).

A estratégia de busca para coleta de dados em ambas as bases, foi feita através do termo "Propriedade Intelectual". Na BDTD, a pesquisa foi realizada em busca avançada, filtrando a opção assunto, tipo de documento e o ano da defesa. A partir de então, foi feito um levantamento nas instituições que fizeram parte da pesquisa. Nos RIs, a busca foi constituída selecionando a comunidade mais conveniente, pois em cada instituição os repositórios possuem comunidades específicas; em seguida, foi escolhido o campo assunto.

Após a coleta dos dados, os mesmos foram organizados no software Microsoft Excel ${ }^{\circledR}$, na qual foram elaboradas as Tabelas e os Gráficos, para visualização dos resultados. As variáveis destacadas para mensuração dos dados foram: Nível de qualificação, Ano de defesa, Instituição, Programa de Pós-Graduação, Palavras-chave. Tais variáveis permitiram construir o perfil da produção científica da Propriedade Intelectual, respondendo aos objetivos propostos nesta pesquisa e a construção do indicador medidas de produção.

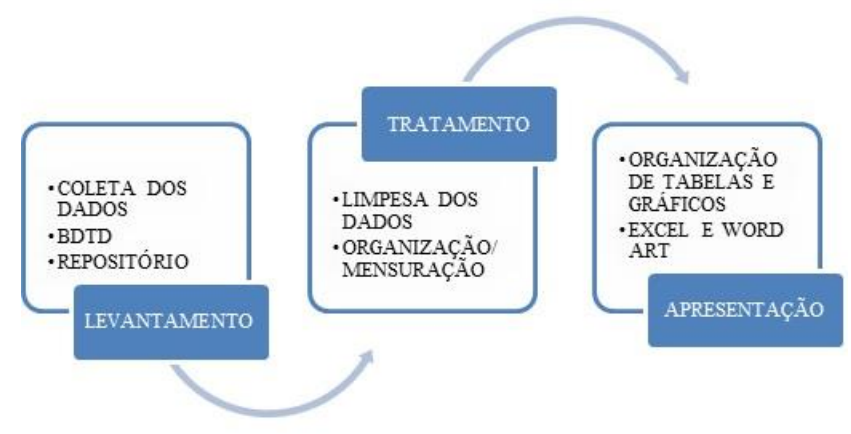

Figura 1. Fluxo do levantamento, tratamento e apresentação dos dados Fonte: Elaboração dos autores. 


\section{Resultados e Discussão}

Nesta seção serão apresentadas as análises bibliométricas dos indicadores de medida de produção, cujas variáveis são: Nível de qualificação, ano de defesa, instituição, Programa de Pós-Graduação e palavras-chave. Os dados foram coletados até fevereiro de 2018, tendo sido encontrados, ao todo, 38 trabalhos. Com base neste levantamento, serão analisadas as variáveis que foram propostas nesta pesquisa, para que se possa delinear e analisar os indicadores bibliométricos da produção científica sobre Propriedade Intelectual.

O Gráfico 1 mostra a distribuição dos trabalhos quanto ao tipo de publicação; foram identificadas quatro teses e 34 dissertações, das quais cinco são provenientes de mestrados profissionalizantes. Este quantitativo permite observar que o mestrado se sobrepõe ao doutorado com relação às pesquisas em Propriedade Intelectual.

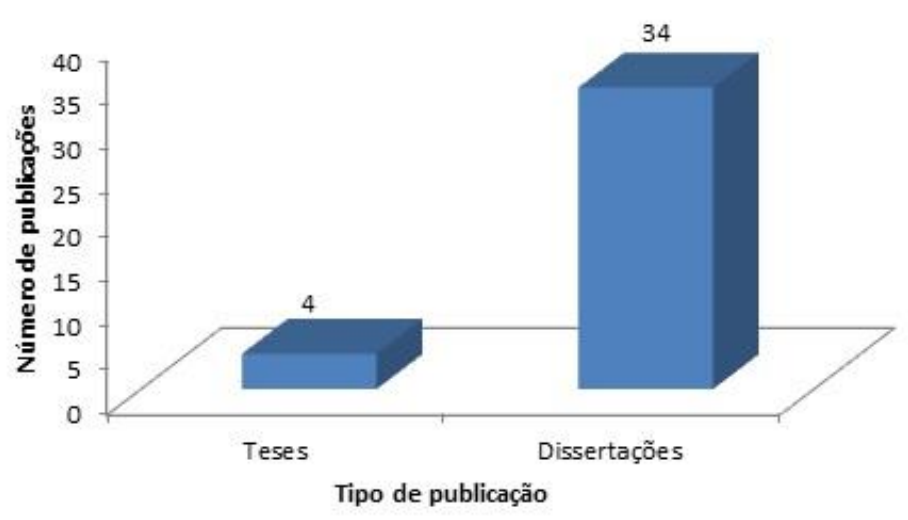

Gráfico 1. Quantitativo por tipo de publicação Fonte: os autores, pesquisa de campo. 


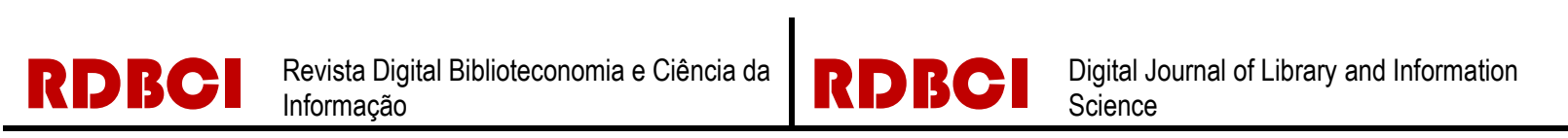

No que diz respeito à distribuição temporal das publicações, no período entre $2005 \mathrm{e}$ 2017, verifica-se, através do Gráfico 2, que as defesas começaram a partir de 2007, com apenas um trabalho. Houve variação quanto ao número de defesas no período analisado; entretanto, a partir de 2014, o quantitativo foi mais elevado. O período com maior número de trabalhos defendidos foi entre os anos de 2014 a 2016. O primeiro trabalho defendido foi uma dissertação de mestrado profissional na UFBA, intitulado: “A proteção e exploração da propriedade intelectual no ambiente universitário: o papel das agências de fomento governamentais".

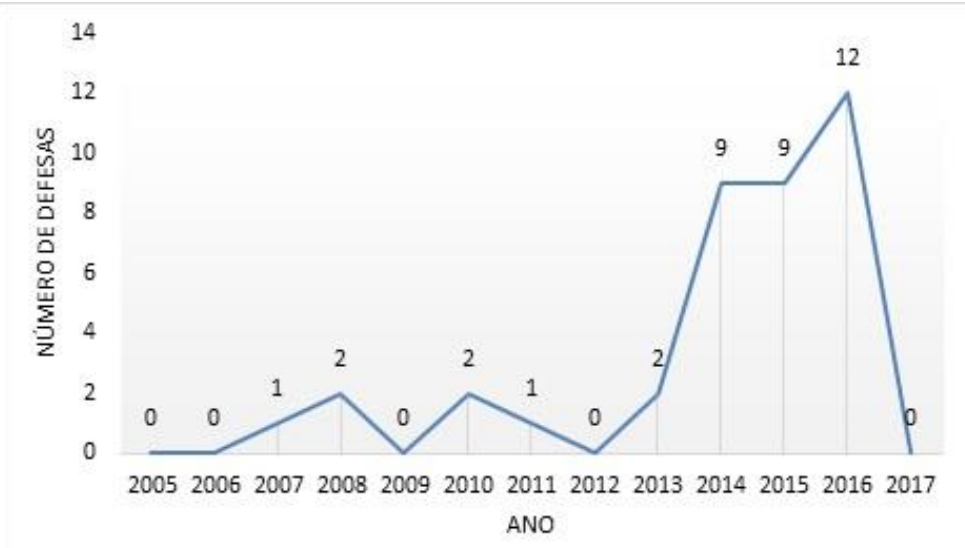

Gráfico 2. Distribuição anual das defesas de Dissertações e de Teses Fonte: os autores, pesquisa de campo.

No que se refere ao quantitativo de pesquisas por instituição, das nove Universidades apresentadas na Figura 5, observa-se que apenas a Universidade Federal do Piauí (UFPI) não possui nenhuma pesquisa voltada ao tema. A Universidade Federal de Sergipe (UFS) detém o destaque, com a maioria das defesas, apresentando 21 trabalhos. Vale salientar que a UFS é uma instituição proeminente pelo fato de oferecer a Pós-Graduação de Ciência da Propriedade Intelectual, em nível de mestrado e de doutorado. Em seguida, destacam-se a UFPB e UFPE, ambas apresentando o mesmo quantitativo, com cinco trabalhos defendidos. A Universidade Federal da Bahia (UFBA) apresenta três trabalhos, as demais Universidades: Universidade Federal de Alagoas (UFAL), Universidade Federal do Ceará (UFC), Universidade Federal do Maranhão (UFMA) e Universidade Federal do Rio Grande do Norte (UFRN) apresentam apenas um trabalho cada.

Vale ressaltar que as pesquisas defendidas na UFS foram realizadas em apenas um Programa, o de Ciência da Propriedade Intelectual, enquanto as pesquisas realizadas na UFPB, três são oriundas do Programa de Ciência da Informação, os outros dois trabalhos são do Programa de Administração. As pesquisas defendidas na UFPE são de Programas distintos, sendo cada pesquisa defendida em um programa diferente: Ciência da Computação, Inovação Terapêutica, Economia, Comércio Exterior e Desenvolvimento e meio Ambiente.

\begin{tabular}{l|c|c|c|c|c|}
\hline (c) RDBCl: Rev. Digit. Bibliotecon. Cienc. Inf. & Campinas, SP & v.17 & $1-23$ & e019007 & 2018 \\
\hline
\end{tabular}




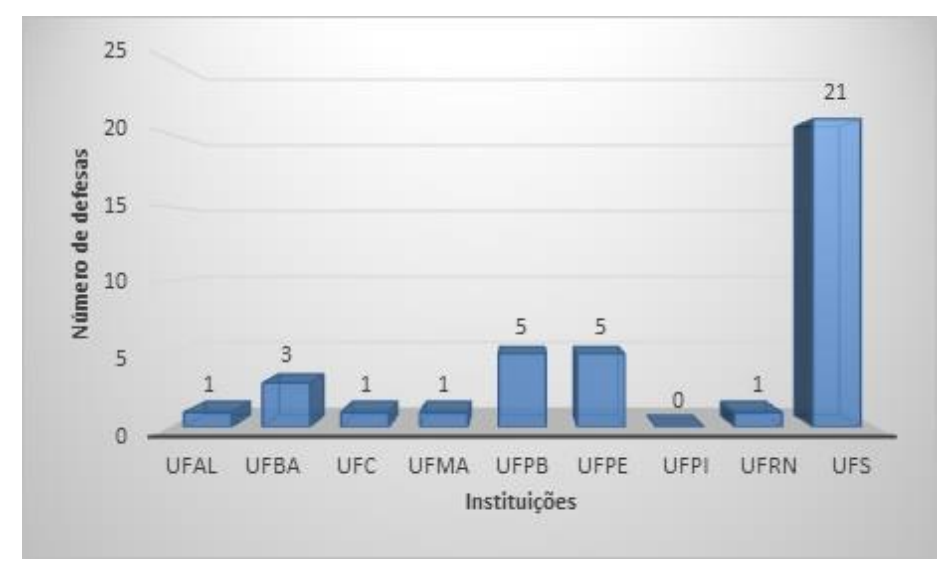

Gráfico 3. Distribuição das defesas por

Universidade Fonte: os autores, pesquisa de campo.

Com relação às pesquisas realizada por Programas de Pós-Graduação, verifica-se, através do Gráfico 4, que 14 programas estão contribuindo com pesquisas, em áreas diversas do conhecimento. De acordo com os dados apresentados, pode-se inferir que a Propriedade Intelectual é tema que desperta interesse em campos distintos do saber, o que permite evidenciar que se trata de uma área interdisciplinar entre as ciências, para a construção de novos conhecimentos.

Além do Programa de Ciência da Propriedade Intelectual, que apresenta 21 trabalhos defendidos, os Programas de Administração e Ciência da Informação se sobressaem aos demais, com três trabalhos cada. Os outros 11 trabalhos foram defendidos em 11 Programas diferentes, conforme se pode inferir no gráfico que se segue.

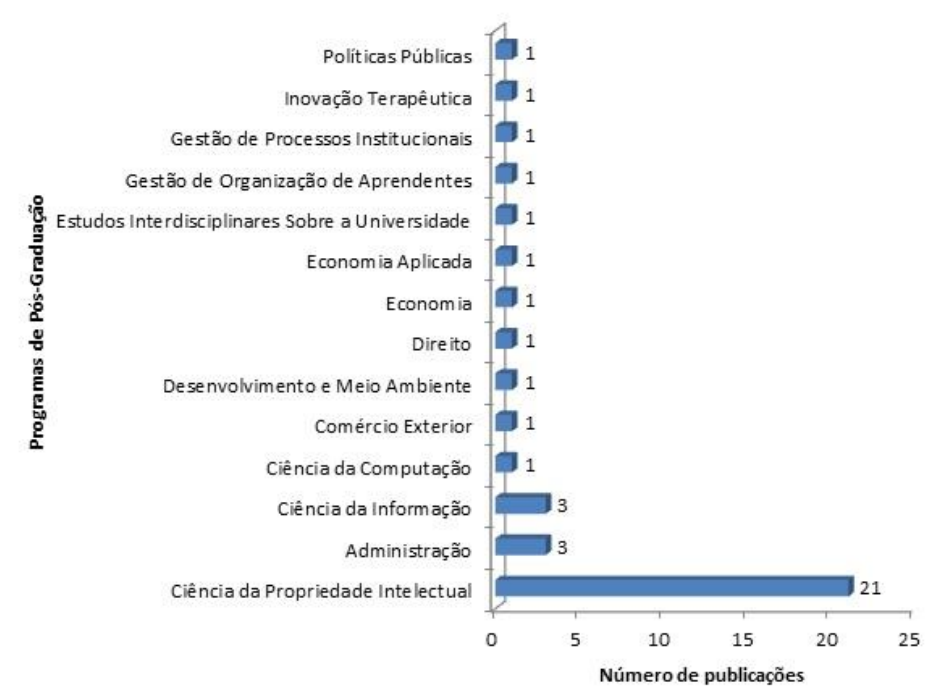

Gráfico 4. Distribuição das defesas por Programa de Pós-Graduação Fonte: os autores, pesquisa de campo. 


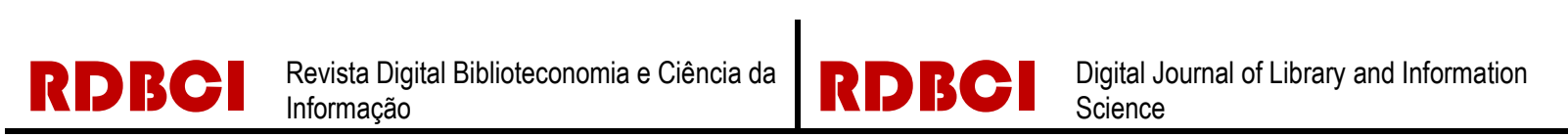

Para melhor ilustrar o que foi exposto anteriormente, sobre a contribuição dos programas com a pesquisa em PI, a Figura 2 mostra a interação da Propriedade Intelectual com os programas que pesquisaram este tema. São 14 os Programas de Pós-Graduação que estão contribuindo para o desenvolvimento da ciência em Propriedade Intelectual.

O mapa expõe uma convergência, na qual todos os programas estão concentrados na periferia, apresentando laços de colaboração de pesquisas com a Propriedade Intelectual, que por sua vez se concentra no centro. É possível perceber a robustez da linha de ligação entre a Ciência da Propriedade Intelectual, o que indica que há mais produção científica neste Programa. Em seguida, percebe-se mais saliência nas linhas em Ciência da Informação e Administração.

Quanto às áreas de conhecimento, verifica-se que metade dos programas apresentados faz parte da área de Ciências Sociais Aplicadas; em segundo lugar, estão os programas provenientes da área de Ciências Humanas.

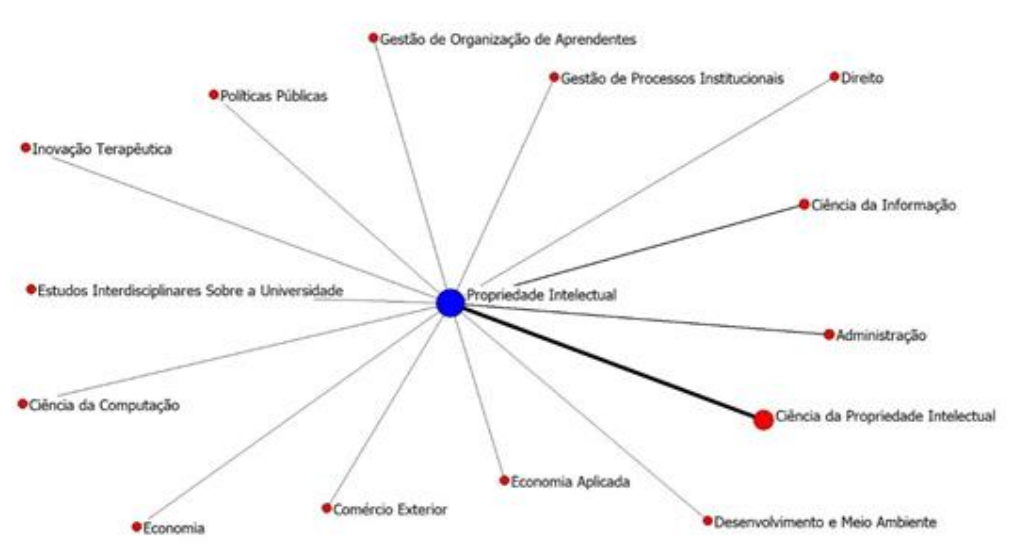

Figura 2. Contribuição dos Programas de Pós-Graduação para a Ciência em Propriedade Intelectual Fonte: os autores, pesquisa de campo.

Com o objetivo de identificar os termos mais recorrentes nas Dissertações, foram avaliadas as palavras-chave utilizadas pelos autores. Nas 34 Dissertações foram encontradas 124 palavras-chave, aproximadamente uma média de 3,6 por trabalho.

Através da Figura 3 verifica-se as palavras-chave nas Dissertações que estão realçadas, ou seja, os termos revelam suas frequências proporcionalmente ao destaque visual. Quanto mais destacado o termo na nuvem, maior a sua frequência. Observa-se que o termo Propriedade Intelectual foi o mais destacado, seguido de Direito autoral, Indicadores, Patentes, Inovação e etc. 


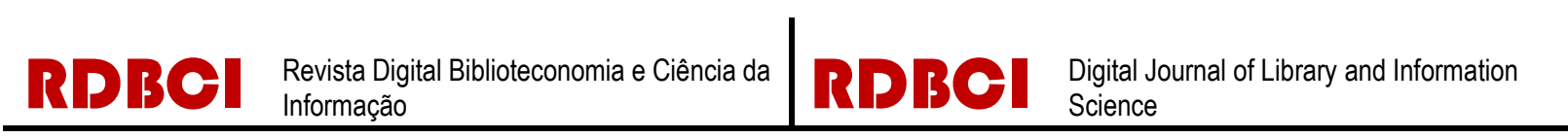

A análise de frequência de ocorrência de palavras está relacionada à Lei de Zipf, que diz respeito à correlação entre a frequência de uma palavra no texto e a posição que ocupa. A posição foi estabelecida por Zipf, em decorrência de uma lista organizada em série, que continha o rank das palavras. As palavras mais citadas recebem a ordem de série um e as demais ordens são classificadas de acordo com a frequência das palavras. Assim, ele observou que o produto da ordem de série de uma palavra pela sua frequência era uma constante (GUEDES, 2012).

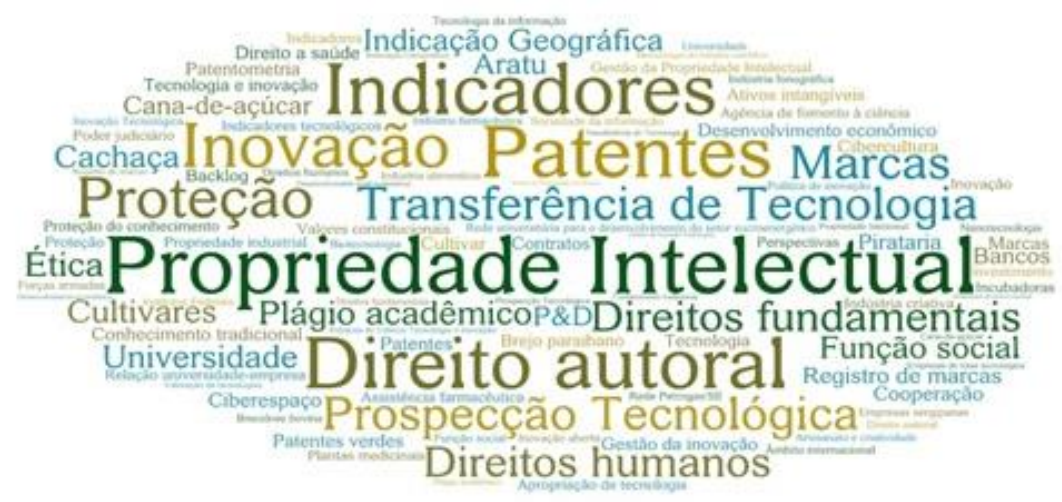

Figura 3. Destaque das palavras-chave das

Dissertações Fonte: os autores, pesquisa de campo.

A Figura 4 apresenta as palavras-chave mais utilizadas pelos autores nas teses. Nas quatro Teses foram encontrados 19 termos, dentre os quais, verifica-se que o maior destaque está em Propriedade Intelectual, seguido de Bens informacionais, Ciência aberta, Software livre e Inovação tecnológica.

As palavras-chave têm como função a representação temática de um documento, ou seja, traduzem um conteúdo, indicam a cobertura de um assunto, além de auxiliar na recuperação da informação, bem como subsidiar a indexação de documentos relacionados. São elementos relevantes para a recuperação e representação da informação no contexto da pesquisa científica (GONÇALVES, 2008). 


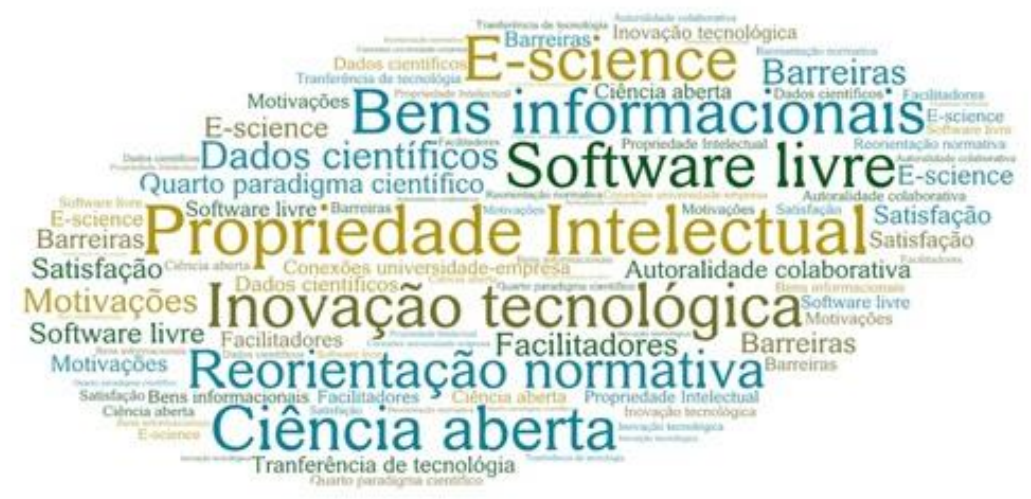

Figura 4. Destaque das palavras-chave das

Teses Fonte: os autores, pesquisa de campo.

Sobre as temáticas abordadas, foram separados em blocos os 21 trabalhos defendidos pelo Programa de Pós-Graduação em Ciência da Propriedade Intelectual, dos quais 20 são de Mestrado (95\%) e um do doutorado (5\%). Foram escolhidos os trabalhos deste programa para análise, devido ao quantitativo ser o maior entre os outros programas. A contribuição das pesquisas neste programa contempla temáticas diversificadas. Entre os temas abordados encontram-se:

a) Pesquisas que abordam o registro de Marcas:

A pesquisa de Medeiros Filho (2014) apresenta a avaliação das empresas sergipanas, no que diz respeito à promoção da proteção do registro de suas marcas. Foram pesquisadas 761 empresas de atuações diferentes, das quais apenas 8,94\% realizaram pedido de registro de marcas.

Almeida (2015) traz também contribuições semelhantes, ao discorrer sobre a proteção marcária de produtos alimentícios de empresas sergipanas. Os dados coletados no INPI apontaram que, das 595 empresas pesquisadas, apenas $12 \%$ realizaram pedidos para o registro de suas marcas.

A pesquisa de Santos (2016) aborda a proteção de marcas do setor bancário no Brasil, o qual propõe verificar a situação da proteção das marcas das agências bancárias públicas e privadas, o quanto essa proteção impacta nos resultados dessas organizações, desde o aumento do valor da sua marca até a atração de novos investidores e na relação de satisfação do cliente. 


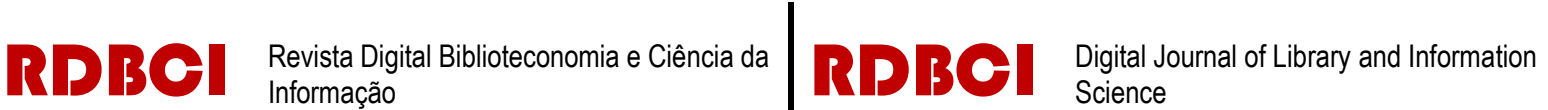

b) Pesquisas voltadas às Patentes e Inovação tecnológica:

Quanto à contribuição de pesquisas sobre patentes e inovação tecnológica, encontra-se na pesquisa de Acioli (2015) o patenteamento das indústrias farmacêuticas no Brasil, cujo objetivo foi analisar os pedidos de patentes depositadas no INPI pelas empresas que lideram o mercado neste setor, bem como analisar os indicadores que categorizam as tendências e estratégias.

Cruz (2016) apresenta um mapeamento dos estudos tecnológicos sobre o uso da nanotecnologia nos tratamentos medicinais, com o objetivo de verificar o crescimento dos depósitos de patentes e as lacunas existentes desta área no Brasil. Constatou que o número de patentes vem se desenvolvendo no Brasil e na Europa.

A pesquisa realizada por Dall'Agnol (2015) apresenta um paralelo da Propriedade Intelectual nas Forças Armadas Brasileiras, Marinha, Exército e Aeronáutica quanto aos depósitos de patentes e as políticas de criação dos seus Núcleos Tecnológicos. Constatou-se que a Força Aérea Brasileira possui o maior número de patentes e o Exército é quem menos deposita.

No texto de Garcez Junior (2015), são avaliados os pedidos de patentes pendentes no INPI e aponta alternativas para diminuição do estoque.

Oliveira (2014) apresenta contribuições através de seu trabalho, cujo objetivo foi realizar um mapeamento tecnológico, para verificar o controle e combate da brucelose bovina, bem como avaliar a erradicação no estado de Sergipe.

\section{c) Pesquisas que abordam a Transferência de tecnologia e PI nas universidades:}

No que diz respeito às pesquisas sobre transferência de tecnologia nas universidades, pode ser visto na Tese de Fabris (2016) as conexões entre empresas e universidades, cujo objetivo foi construir e validar um modelo para analisar como e porque ocorrem as conexões Empresa-Universidade.

Já na pesquisa de Moura (2015), foi analisado como funciona a proteção da Propriedade Intelectual nas instituições de ensino superior na região Nordeste, mais especificamente se existem e como funcionam os contratos de Propriedade Intelectual.

Na pesquisa de Pires (2016) desenvolveu-se um estudo de caso na Universidade Federal do Recôncavo da Bahia, tendo como objetivo mapear e propor estratégias para

\begin{tabular}{l|c|c|c|c|c}
\hline (c) RDBCl: Rev. Digit. Bibliotecon. Cienc. Inf. & Campinas, SP & v.17 & $1-23$ & e019007 & 2018
\end{tabular}




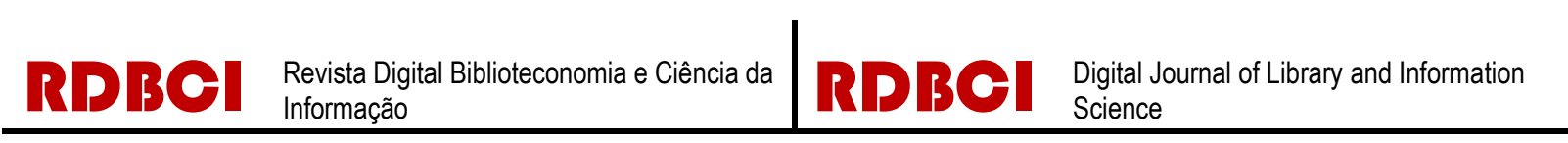

intensificar a proteção da Propriedade Intelectual e a transferência de tecnologia.

Tem-se em Souza (2016), modelos de valoração de patentes, cujo objetivo foi investigar as tendências de pesquisa e os métodos de valoração de patentes nas mais importantes universidades públicas brasileiras, com o intuito de demonstrar o desempenho das universidades quanto ao depósito de pedido de patentes.

Outros temas foram abordados nas Dissertações, que se somam como contribuição à área da Propriedade Intelectual: Ensino da PI, desenvolvido por Tommasi (2015); sobre Indicação geográfica temos a Dissertação de Brandão (2016); a Mensuração da produção científica pode ser vista na pesquisa desenvolvida por Menezes (2016); Aspectos sobre a função social da PI pode ser observado na pesquisa de Lima (2016); a Gestão da Propriedade Intelectual foi tema das Dissertações de Silva (2014) e Loureiro (2016); sobre Cultivares, temos a contribuição da pesquisa de Borges (2014).

\section{Considerações Finais}

De acordo com os objetivos propostos e com base nos resultados apresentados, evidencia-se que a Propriedade Intelectual tem sido tema de pesquisas nas Universidades Federais da Região Nordeste.

Com relação às variáveis que constituíram o indicador medidas de produção, após a análise bibliométrica, tem-se quanto ao nível acadêmico, o mestrado como predominante nas pesquisas sobre Propriedade Intelectual, com 34 trabalhos, enquanto o doutorado apresentou quatro pesquisas. O período em que houve mais defesas foi de 2014 a 2016.

No que diz respeito às pesquisas por instituição, destaca-se a Universidade Federal de Sergipe com 21 trabalhos, representando 55,3\% do quantitativo de 38, entre Dissertações e Teses, dado que projeta o Estado de Sergipe em primeiro lugar na Região Nordeste.

Quanto aos Programas de Pós-Graduação, dos 14 apresentados o Programa de PósGraduação em Ciência da Propriedade Intelectual, da Universidade Federal de Sergipe foi o mais proeminente nas pesquisas sobre o tema, seguido dos Programas em Ciência da Informação e Administração.

No que diz respeito às temáticas abordadas, Programas de diferentes áreas do conhecimento estão contribuindo para a ciência em Propriedade Intelectual, podendo-se inferir que há uma interação interdisciplinar entre as áreas. Vale destacar as temáticas mais 


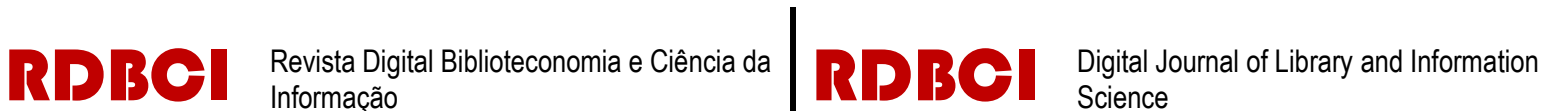

abordadas: Registro de marcas; Patentes e Inovação Tecnológica; Transferência de Tecnologia e PI nas Universidades; Direito autoral e Indicação Geográfica.

A análise de frequência das palavras-chave mostrou que o termo mais recorrente foi Propriedade Intelectual, tanto nas Teses como nas Dissertações; outros termos mais utilizados foram: Patentes, Inovação e Direito autoral.

Este estudo abre perspectivas para diversas análises, de forma que se possa identificar mais indicadores com a possibilidade de explorar outros caminhos da pesquisa em Propriedade Intelectual. Tais indicadores permitirão apontar contribuições no campo da Propriedade Intelectual para a ciência.

\section{Referência}

ALVAREZ, Gonzalo Rubén; CAREGNATO. Sônia Elisa. A ciência da informação e sua contribuição para a avaliação do conhecimento científico. Biblos: Revista do Instituto de Ciências Humanas e da Informação, v. 31, n. 1, p. 9-26, jan./jun. 2017. Disponível em: http://periodicos.ufpb.br/index.php/pbcib/article/view/36565. Acesso em: 01 fev. 2018.

ARAÚJO, Carlos A. Bibliometria: evolução histórica e questões atuais. Em questão. Porto Alegre, v. 12, n. 1, 2006.

BRASIL. Lei $\mathbf{n}^{0}$ 9.610/1998. Altera, atualiza e consolida a legislação sobre direitos autorais e dá outras providências. Brasília (DF): Congresso Nacional; 1998.

CAMPOS, Antônio Carlos de; DENIG, Edmila Adriana. Propriedade intelectual: uma análise a partir da evolução das patentes no Brasil. Revista Faz Ciência. v. 13, n. 18, jul./dez. 2011, p. 97-120. Disponível em: http://e-revista.unioeste.br/index.php/fazciencia/article/ view/7977/6700. Acesso em: 27 jun. 2018.

CUSTÓDIO, Pollyana Ágata Gomes da Rocha. Dissertações e teses da pós-graduação em educação na Unesp-Campus de Marília: um estudo das citações e cocitações (2004 a 2009). 2012. 145 f. Dissertação (mestrado) - Faculdade de Filosofia e Ciências de Marília, Universidade Estadual Paulista Júlio de Mesquita Filho, 2012. Disponível em: http://hdl.handle.net/11449/93676. Acesso em: 09 out. 2017.

GONÇALVES, Aline Lima. Uso de resumos e palavras-chave em Ciências Sociais: uma avaliação. Encontros Bibli: Revista Eletrônica de Biblioteconomia e Ciência da Informação. Florianópolis, v. 2, n. 26, 2008. Disponível em https://periodicos.ufsc.br/index.php/eb/issue/archive. Acesso em: 23 abr. 2018.

GORBEA PORTAL, Salvador. Una nueva perspectiva teórica de la bibliometría basada em su dimensión histórica y sus referentes temporales. Investig. bibl, México, v. 30, n. 70, p. 11-

\begin{tabular}{l|l|l|l|l|l|}
\hline (c) RDBCl: Rev. Digit. Bibliotecon. Cienc. Inf. & Campinas, SP & v.17 & $1-23$ & e019007 & 2018 \\
\hline
\end{tabular}




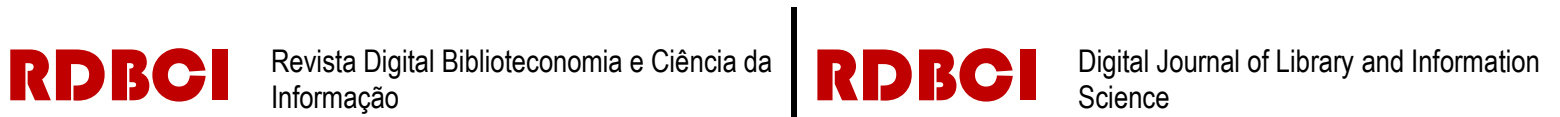

16, 2016 . Disponível em

http://www.scielo.org.mx/scielo.php?script=sci_arttext\&pid=S0187-

$358 \mathrm{X} 2016000300011 \& \operatorname{lng}=\mathrm{es} \& \mathrm{nrm}=\mathrm{iso}$. Acesso em:

24 abr. 2018. http://dx.doi.org/10.1016/j.ibbai.2016.10.001.

GUEDES, V. L. da S. A bibliometria e a gestão da informação e do conhecimento científico e tecnológico: uma revisão da literatura. Ponto de Acesso, Salvador, v.6, n. 2, p. 74-109 ago.

2012. Disponível em: http://www.portalseer.ufba.br/index.php/

revistaici/article/viewArticle/5695. Acesso em: 12 fev. 2018.

http://www.scielo.br/pdf/ci/v33n2/a13v33n2.pdf. Acesso em: 08 fev. 2018.

IBCT, Instituto Brasileiro de Informação em Ciência e Tecnologia. Repositórios

digitais. Disponível em: http://www.ibict.br/informacao-para-ciencia-tecnologia-einovacao\%20/repositorios-digitais/historico. Acesso em: 14 jan. 2018.

IBCT, Instituto Brasileiro de Informação em Ciência e Tecnologia. Biblioteca Digital de Teses e Dissertações. Disponível em: http://www.ibict.br/informacao-para-cienciatecnologia-e-inovacao\%20/repositorios-digitais/historico. Acesso em: 14 jan. 2018.

JUNGMANN, Diana de Mello; BONETTI, Esther Aquemi. Inovação e propriedade intelectual: guia para o docente. Brasília: SENAI, 2010. Disponível em: http://www.inpi.gov.br/sobre/arquivos/guia_docente_iel-senai-e-inpi.pdf. Acesso em: 17 out. 2017.

LOPES, Sílvia et al. A bibliometria e a avaliação da produção científica: indicadores e ferramentas. In: ACTAS DOS CONGRESSOS NACIONAIS DE BIBLIOTECÁRIOS, ARQUIVISTAS E DOCUMENTALISTAS, 11., 2012. Disponível em:

http://www.bad.pt/publicacoes/index.php/congressosbad/issue/view/10. Acesso em: 07 nov. 2017.

MARCELO, Júlia Fernandes; HAYASHI, Maria Cristina Piumbato Innocentini. Estudo bibliométrico sobre a produção científica no campo da sociologia da ciência. Informação \& Informação, Londrina, v. 18, n. 3, p. 138 - 153, set./dez. 2013. Disponível em: http://www.uel.br/revistas/uel/index.php/informacao/article/view/8413/pdf_2. Acesso em: 06 fev. 2018.

MUGNAINI, R. Produção e impacto da pesquisa brasileira: confrontando contextos internacional e nacional. SciELO em Perspectiva, 2018. Disponível em: https://blog.scielo.org/blog/2018/08/27/producao-e-impacto-da-pesquisa-brasileiraconfrontando-contextos-internacional-e-nacional/\#.W_8prNtKiUk. Acesso em: 28 nov. 2018.

PIMENTA, Alcineide Aguiar et al. A bibliometria nas pesquisas acadêmicas. SCIENTIA:

\begin{tabular}{l|c|c|c|c|c|}
\hline (c) RDBCl: Rev. Digit. Bibliotecon. Cienc. Inf. & Campinas, SP & v.17 & $1-23$ & e019007 & 2018 \\
\hline
\end{tabular}




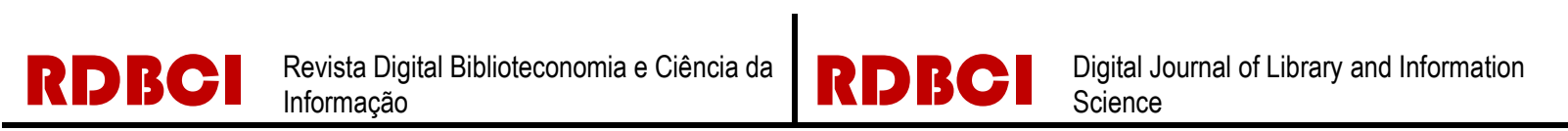

Revista de ensino, pesquisa e extensão, Faculdade Luciano Feijão. v. 4, n. 7, 2017. Disponível em: http://flucianofeijao.com.br. Acesso em: 20. abr. 2018.

SPEZIALI, Marcelo Gomes et al. Cartilha de propriedade intelectual. 2016.

Disponível em: http://saci2.ufop.br/data/solicitacao/08749_cartilha_nite_versao_final.pdf. Acesso em: 08 nov. 2017.

\section{UNIVERSIDADE FEDERAL DE SERGIPE (UFS). Cartilha UFS de propriedade} intelectual. São Cristóvão, SE, 2017. 50 p. Disponível em:

http://cinttec.ufs.br/uploads/page_attach/path/3517/Cartilha_Propriedade_Intelectual_Digita 1.pdf. Acesso em: 28 nov. 2018.

WIPO. Global network on intellectual property (IP) academies. 2016. Disponível em: http://www.wipo.int/academy/en/about/global_network/. Acesso em: 30 nov. 2017.

\section{APÊNDICE A -}

Referências das Teses que fizeram parte da pesquisa

FABRIS, Jonas Pedro. Conexões entre empresas e universidades. 2016. $117 \mathrm{f}$. Tese (Doutorado em Ciência da Propriedade Intelectual) - Programa de Pós-Graduação em Ciência da Propriedade Intelectual, Universidade Federal de Sergipe, 2016. Orientadora: Profa. Dra. Maria Emilia Camargo.

OLIVEIRA, Adriana Carla Silva de. Desvendando a autoralidade colaborativa na escience sob a ótica dos direitos de propriedade intelectual. 2016. $299 \mathrm{f}$. Tese (Doutorado em Ciência da Informação) - Centro de Ciências Sociais Aplicadas, Universidade Federal da Paraíba, 2016. Orientador: Prof. Dr. Guilherme Ataide Dias.

SOARES, Danielle de Queiroz. Inovação tecnológica e propriedade intelectual no capitalismo contemporâneo: o contraditório caso do software livre. 2015. $214 \mathrm{f}$. Tese (Doutorado em Políticas Públicas) - Centro de Ciências Sociais, Universidade Federal do Maranhão, São Luis, 2015. Orientador: Prof. Dr. Flávio Bezerra de Farias.

SOUSA, Rosilene Paiva Marinho de. A informação como elemento de regulação dos institutos jurídicos da propriedade intelectual. 2016. $126 \mathrm{f}$. Tese (Doutorado em Ciência da Informação) - Programa de Pós-Graduação em Ciência da Informação, Universidade Federal da Paraíba, João Pessoa, 2016. Orientador: Prof. Dr. Guilherme Ataide Dias. 


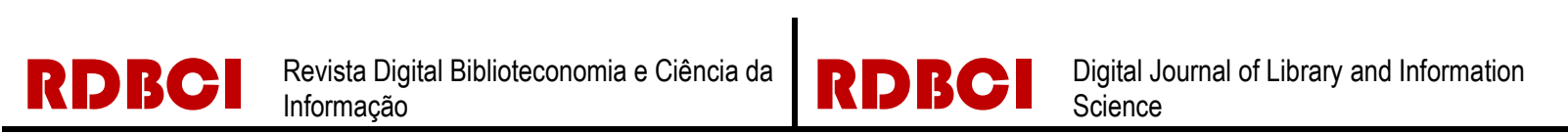

\author{
APÊNDICE B - \\ Referências das Dissertações que fizeram parte da pesquisa
}

ACIOLI, Gláucia da Rocha. Patenteamento na indústria farmacêutica no Brasil : tendências, oportunidades e estratégias. 2015. 90 f. Dissertação (Mestrado em Ciência da Propriedade Intelectual) - Programa de Pós-Graduação em Ciência da Propriedade Intelectual, Universidade Federal de Sergipe, São Cristóvão, 2015. Orientador: Prof. Dr. Antônio Martins de Oliveira Junior.

ALMEIDA, Glessiane de Oliveira. Proteção marcária relacionada aos produtos alimentícios de empresas do estado de Sergipe. 2015. 55 f. Dissertação (Mestrado em Ciência da Propriedade Intelectual) - Programa de Pós-Graduação em Ciência da Propriedade Intelectual, Universidade Federal de Sergipe, São Cristóvão, 2015. Orientadora: Profa. Dra. Elma Regina Silva de Andrade Wartha.

BORGES, Sayonara Marinho Soares. PI de cultivares na Embrapa Tabuleiros Costeiros : estudo das relações de parceria. 2014. 135 f. Dissertação (Mestrado em Ciência da Propriedade Intelectual) - Programa de Pós-Graduação em Ciência da Propriedade Intelectual, Universidade Federal de Sergipe, São Cristóvão, 2014. Orientador: Prof. Dr. Gabriel Francisco da Silva.

BRANDÃO, Bárbara de Oliveira. A valorização dos produtos tradicionais através da indicação geográfica: o potencial do aratu de Santa Luzia do Itanhy. 2016. 74 f. Dissertação (Mestrado em Ciência da Propriedade Intelectual) - Programa de Pós-Graduação em Ciência da Propriedade Intelectual, Universidade Federal de Sergipe, São Cristóvão, 2015. Orientador: Prof. Dr. João Antonio Belmino dos Santos.

CRUZ, Cleide Ane Barbosa da. Inovação tecnológica: um mapeamento de patentes sobre o uso da nanotecnologia em diagnósticos e tratamentos médicos. 2016. 69 f. Dissertação (Mestrado em Ciência da Propriedade Intelectual) - Programa de Pós-Graduação em Ciência da Propriedade Intelectual, Universidade Federal de Sergipe, São Cristóvão, 2016.

Orientadora: Profa. Dra. Ana Eleonora Almeida Paixão. 


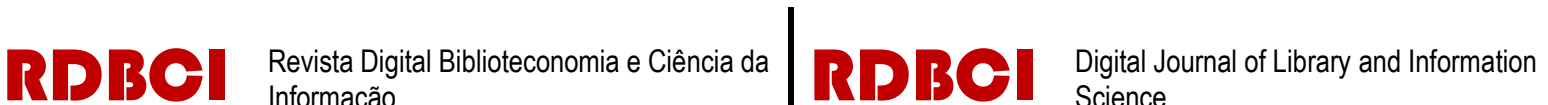

\section{DALL'AGNOL, Rogéria Prado. A propriedade intelectual nas Forças Armadas}

Brasileiras: um paralelo entre a Marinha, Exército e Aeronáutico quanto aos depósitos de patentes e as políticas de criação dos NITs. 2015. 73 f. Dissertação (Mestrado em Ciência da Propriedade Intelectual) - Programa de Pós-Graduação em Ciência da Propriedade Intelectual, Universidade Federal de Sergipe, São Cristóvão, 2015. Orientador: Prof. Dr. Glaucio José Couri Machado.

GARCEZ JUNIOR, Silvio Sobral. A evolução de pedidos de patente com análise pendente no INPI: construindo alternativas para proteção do depositante e diminuição do backlog. 2015. 108 f. Dissertação (Mestrado em Ciência da Propriedade Intelectual) Programa de Pós-Graduação em Ciência da Propriedade Intelectual, Universidade Federal de Sergipe, São Cristóvão, 2015. Orientadora: Profa. Dra. Jane de Jesus da Silveira Moreira.

JESUS, Patrícia Castro de. Apropriação do conhecimento gerado na UFBA visando transferência de tecnologia (TT) para a sociedade. 2014. 93 f. Dissertação (Mestrado em Estudos Interdisciplinares sobre a Universidade) - Instituto de Humanidades, Artes e Ciências, Universidade Federal da Bahia, Salvador, 2014. Orientador: Prof. Dr. Luiz Alberto Luz de Almeida.

LIMA, Joelma dos Santos. Função social da propriedade intelectual: aspectos gerais e perspectivas. 2016. 96 f. Dissertação (Mestrado em Ciência da Propriedade Intelectual) Programa de Pós-Graduação em Ciência da Propriedade Intelectual, Universidade Federal de Sergipe, São Cristóvão, 2016. Orientador: Prof. Dr. Daniel Pereira da Silva.

LOUREIRO, Rodrigo Nogueira Albert. A gestão da propriedade intelectual nos institutos federais: ações para construção de uma cultura de propriedade intelectual no âmbito do Instituto Federal de Educação, Ciência e Tecnologia de Pernambuco. 2016. 101 f. Dissertação (Mestrado em Ciência da Propriedade Intelectual) - Programa de Pós-Graduação em Ciência da Propriedade Intelectual, Universidade Federal de Sergipe, São Cristóvão, 2016. Orientador: Prof. Dr. Glaucio José Couri Machado.

MASCARENHAS, Tatiane Cordeiro. Gestão de ativos de propriedade intelectual: práticas adotadas por empresas na Bahia. 2008. $221 \mathrm{f}$. Dissertação (mestrado em Administração) - Escola de Administração, Universidade Federal da Bahia, Salvador, 2008. Orientadora: Profa. Dr. ${ }^{a}$ Elisabeth Regina Loiola da Cruz Souza.

MEDEIROS FILHO, Adonis Reis de. Registro de marca: o uso do sistema marcário por empresas sergipanas. 2014. 68 f. Dissertação (Mestrado em Ciência da Propriedade Intelectual) - Programa de Pós-Graduação em Ciência da Propriedade Intelectual, Universidade Federal de Sergipe, São Cristóvão, 2014. Orientadora: Profa. Dra. Suzana Leitão Russo.

MENEZES, Edmara Thays Neres. Mensuração da produção científica e tecnológica de pesquisadores da Universidade Federal de Sergipe após a Lei de Inovação. 2016. 72 f. Dissertação (Mestrado em Ciência da Propriedade Intelectual) - Programa de Pós-Graduação em Ciência da Propriedade Intelectual, Universidade Federal de Sergipe, São Cristóvão, 2016. Orientadora: Profa. Dra. Suzana Leitão Russo. 
MORAIS, Sara Maria Peres de. Prospecção tecnológica em documentos de patentes verdes. 2014. 109 f. Dissertação (Mestrado em Ciência da Informação) - Programa de PósGraduação em Ciência da Informação, Universidade Federal da Paraíba, João Pessoa, 2014. Orientadora: Profa. Dra. Joana Coeli Ribeiro Garcia.

MOURA, Sâmia Passos Barboza. Contratos em propriedade intelectual: a utilização nas instituições de ensino superior nordestinas. 2015. 119 f. Dissertação (Mestrado em Ciência da Propriedade Intelectual) - Programa de Pós-Graduação em Ciência da Propriedade Intelectual, Universidade Federal de Sergipe, São Cristóvão, 2015. Orientadora: Profa. Dra. Suzana Leitão Russo.

NUNES, Fernando José de Holanda. Do long-play ao mp3: indústria fonográfica brasileira na era digital. 2013. 99 f. Dissertação (Mestrado em Economia) - Programa de PósGraduação em Economia, Universidade Federal de Pernambuco, Recife, 2013. Orientador: Prof. Dr. João Policarpo Rodrigues Lima.

NÚÑEZ, Maria Fabiana Perez Durán. A proteção e exploração da propriedade intelectual no ambiente universitário: o papel das agências de fomento governamentais. 2007. $143 \mathrm{f}$. Dissertação (Mestrado Profissional em Administração) - Escola de Administração, Universidade Federal da Bahia, Salvador, 2007. Orientadora: Profa. Dra. Mônica de Aguiar Mac-Allister da Silva.

OLIVEIRA, José Glaucio Brito Tavares de. Autoria e plágio em trabalhos acadêmicos: Políticas e ações de combate a contrafação no contexto universitário. 2016. 67 f. Dissertação (Mestrado Profissional em Gestão de Processos Institucionais) - Programa de Pós-Graduação em Gestão de Processos Institucionais, Universidade Federal do Rio Grande do Norte, Natal, 2016. Orientador: Sérgio Luís Rizzo Dela-Sávia.

OLIVEIRA, Luana Brito de. Mapeamento tecnológico sobre o controle e combate da brucelose bovina. 2014. 59 f. Dissertação (Mestrado em Ciência da Propriedade Intelectual) - Programa de Pós-Graduação em Ciência da Propriedade Intelectual, Universidade Federal de Sergipe, São Cristóvão, 2014. Orientadora: Profa. Dra. Maria Emília Camargo.

PEREIRA, Maísa Cavalcante. Acesso a medicamentos no SUS: observações sobre decisões jurídicas, políticas e econômicas a partir de demandas junto ao Tribunal de Justiça de Pernambuco. 2011. 115 f. Dissertação (Mestrado em Inovação Terapêutica) - Programa de Pós-Graduação em Inovação Terapêutica, Universidade Federal de Pernambuco, Recife, 2011. Orientador: Prof. Dr. Artur Stamford da Silva.

PIRES, Edilson Araújo. Mapeamento das estratégias para intensificar a proteção da propriedade intelectual e a transferência de tecnologia: um estudo de caso da Universidade Federal do Recôncavo da Bahia. 2014. 130 f. Dissertação (Mestrado em Ciência da Propriedade Intelectual) - Programa de Pós-Graduação em Ciência da Propriedade Intelectual, Universidade Federal de Sergipe, São Cristóvão, 2016. Orientadora: Profa. Dra. Cristina Maria Assis Lopes Tavares da Mata Hermida Quintela. 


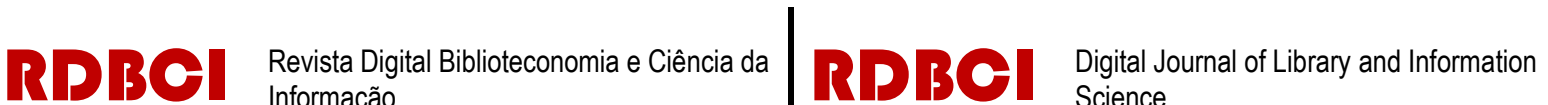

ROCHA, Afonso de Paula Pinheiro. Propriedade intelectual e suas implicações constitucionais: análise do perfil constitucional da propriedade intelectual e suas interrelações com valores constitucionais e direitos fundamentais. 2008. 287 f. Dissertação (Mestrado em Direito) - Faculdade de Direito, Universidade Federal do Ceará, Fortaleza, 2008. Orientador: Prof. João Luis Nogueira Matias.

ROCHA, Elaine de Lima. Propriedade intelectual por indicação geográfica: o caso da cachaça do Brejo paraibano. 2016. 132 f. Dissertação (Mestrado em Administração) Programa de Pós-Graduação em Administração, Universidade Federal da Paraíba, João Pessoa, 2016. Orientador: Prof. Dr. Guilherme Ataide Dias.

SANTOS FILHO, Severino Miguel dos. Propriedade intelectual na relação sociedadenatureza: apropriação de conhecimentos oriundos de comunidades que utilizam práticas tradicionais relativas a plantas medicinais. 2010. 92 f. Dissertação (Mestrado em Desenvolvimento e Meio Ambiente) - Programa de Pós-Graduação em Desenvolvimento e Meio Ambiente, Universidade Federal de Pernambuco, Recife, 2010. Orientadora: Profa. Dra. Christiane P. Y. Rufino Dabat.

SANTOS, Ana Paula Silva dos. O mapeamento da indústria criativa sergipana: sua relação com a propriedade intelectual. 2015. 71 f. Dissertação (Mestrado em Ciência da Propriedade Intelectual) - Programa de Pós-Graduação em Ciência da Propriedade Intelectual, Universidade Federal de Sergipe, São Cristóvão, 2015. Orientadora: Profa. Dra. Suzana Leitão Russo.

SANTOS, Rosa Elaine Andrade. Mapeamento da proteção das marcas do setor bancário no Brasil. 2016. 106 f. Dissertação (Mestrado em Ciência da Propriedade Intelectual) Programa de Pós-Graduação em Ciência da Propriedade Intelectual, Universidade Federal de Sergipe, São Cristóvão, 2016. Orientador: Prof. Dr. Gabriel Francisco da Silva.

SANTOS, Maraiza Santana dos. Geração de propriedade intelectual nas empresas da Rede Petrogas de Sergipe. 2015. 71 f. Dissertação (Mestrado em Ciência da Propriedade Intelectual) - Programa de Pós-Graduação em Ciência da Propriedade Intelectual, Universidade Federal de Sergipe, São Cristóvão, 2015. Orientadora: Profa. Dra. Ana Eleonora Almeida Paixão.

SARAIVA, Raquel Lima. "Acesso à informação versus direito de autor: a busca do equilíbrio no contexto da cultura digital”. 2014. 102 f. Dissertação (Mestrado Ciência da Computação) - Universidade Federal de Pernambuco, Recife, 2014. Orientador: Prof. Dr. Ruy José Guerra Barretto de Queiroz.

SILVA, Gracemerce Camboim Jatobá e. Propriedade intelectual e o comercio internacional: a proteção da propriedade intelectual como um dos determinantes-chave para o crescimento. 2010. 67 f. Dissertação (Mestrado Profissional em Comércio Exterior) Programa de Pós-Graduação em Economia, Universidade Federal de uco, Recife, 2010. Orientador: Prof. Dr. Álvaro Hildalgo.

SILVA, Patrícia Brandão Barbosa da. O setor sucroenergético e o regime de apropriabilidade de cultivares de cana-de-açúcar: caso RIDESA. 2013. 97 f. Dissertação (Mestrado em Economia) -- Faculdade de Economia, Administração e Contabilidade, Universidade Federal de Alagoas, Maceió, 2013. Orientador: Prof. Dr. JosealdoTonholo. 
SILVA, Rafaela. Gestão da propriedade intelectual em empresas de base tecnológicas vinculadas à incubadora tecnológica de Campina Grande - PB. 2014. 69 f. Dissertação (Mestrado em Ciência da Propriedade Intelectual) - Programa de Pós-Graduação em Ciência da Propriedade Intelectual, Universidade Federal de Sergipe, São Cristóvão, 2014. Orientador: Prof. Dr. João Antonio Belmino dos Santos.

SOARES, Ronaldo. Proteção à autoria e combate ao plágio acadêmico na FAINOR: referenciais didáticos instrucionais. 2014. 90 f. Dissertação (Mestrado Profissional em Gestão em Organizações Aprendentes) - Centro de Educação, Universidade Federal da Paraíba, João Pessoa, 2014. Orientador: Prof. Dr. Guilherme Ataide Dias.

SOUZA, Pedro Mendes de. Modelos de valoração da propriedade intelectual como indutor de transferência de tecnologia em universidades públicas. 2016. 72 f. Dissertação (Mestrado em Ciência da Propriedade Intelectual) - Programa de Pós-Graduação em Ciência da Propriedade Intelectual, Universidade Federal de Sergipe, São Cristóvão, 2016. Orientador: Prof. Dr. Antônio Martins de Oliveira Junior.

TOMMASI, Alexandre Cardoso. O ensino de propriedade intelectual para o fortalecimento do Sistema Nacional de Informação. 2015. 78 f. Dissertação (Mestrado em Ciência da Propriedade Intelectual) - Programa de Pós-Graduação em Ciência da Propriedade Intelectual, Universidade Federal de Sergipe, São Cristóvão, 2015. Orientador: Prof. Dr. Antônio Martins de Oliveira Junior. 